\title{
Report of the Asian Forum of Chronic Kidney Disease Initiative (AFCKDI) 2007. "Current status and perspective of CKD in Asia": diversity and specificity among Asian countries
}

\author{
Yusuke Tsukamoto $\cdot$ HaiYan Wang $\cdot$ Gavin Becker $\cdot$ Hung-Chun Chen · Dae-Suk Han • \\ David Harris · Enyu Imai · Vivekanand Jha · Philip K. T. Li · Evan J. C. Lee · Seiichi Matsuo • \\ Yasuhiko Tomino $\cdot$ Kriang Tungsanga $\cdot$ Kunihiro Yamagata $\cdot$ Akira Hishida
}

Received: 3 October 2008/Accepted: 18 December 2008/Published online: 14 March 2009

(C) Japanese Society of Nephrology 2009

\begin{abstract}
The Japanese Society of Nephrology (JSN) sponsored the Asian Forum of CKD Initiative (AFCKDI) 2007 with the support of the International Society of Nephrology-Commission for Global Advancement in Nephrology (ISN-COMGAN), Asian Pacific Society of Nephrology (APSN), the Kidney Disease: Improving Global Outcome (KDIGO) and other national societies of
\end{abstract}

\section{Y. Tsukamoto $(\square)$}

Department of Nephrology, Shuwa General Hospital,

Saitama, Japan

e-mail: tsukamoto@jinzou.net

\section{H. Wang}

Division of Nephrology and Institute of Nephrology,

The First Hospital, Peking University,

Beijing, People's Republic of China

\section{G. Becker}

Department of Nephrology, Royal Melbourne Hospital,

Melbourne, VIC, Australia

H.-C. Chen

Department of Nephrology, Kaohsiung Medical University

Hospital, Kaohsiung, Taiwan

\section{D.-S. Han}

Division of Nephrology, Yonsei University College of Medicine, Seoul, Korea

\section{Harris}

Department of Renal Medicine, Westmead Hospital,

University of Sydney, Sydney, NSW, Australia

E. Imai

Department of Nephrology, Osaka University Hospital,

Osaka, Japan

V. Jha

Department of Nephrology, Postgraduate Institute of Medical

Education and Research, Chandigarh, India nephrology in the Asian Pacific region on 27-28 May 2007 in Hamamatsu City, Japan. An international organising committee was established by leading experts of the CKD initiative. The main objective of this forum was to clarify the current status and perspectives of CKD and to promote coordination, collaboration and integration of initiatives in the Asian Pacific region. The forum received 56 papers

\section{P. K. T. Li}

Department of Medicine and Therapeutics,

Prince of Wales Hospital,

The Chinese University of Hong Kong,

Shatin, Hong Kong

\section{E. J. C. Lee}

Department of Medicine, Yong Loo Lin School of Medicine, National University of Singapore, Singapore, Singapore

\section{S. Matsuo}

Department of Nephrology,

Nagoya University School of Medicine,

Nagoya, Japan

Y. Tomino

Department of Medicine, Juntendo University School

of Medicine, Tokyo, Japan

K. Tungsanga

Department of Medicine,

King Chulalongkorn Memorial Hospital,

Bangkok, Thailand

K. Yamagata

Department of Medicine, Tsukuba University School of Medicine, Ibaraki, Japan

\section{A. Hishida}

Department of Nephrology, Hamamatsu Medical University, Shizuoka, Japan 
from 16 countries; it began with the symposium "A Challenge to CKD in the world" and was followed by the ISN-COMGAN affiliated workshop "Current status and perspective of CKD in Asia". The second day was dedicated to discussion on the evaluation, surveillance and intervention in CKD in this area. At the end of the forum, we decided on the future plan as follows: (1) The AFCKDI will provide opportunities annually or biannually for every person who promotes CKD initiatives in the Asian Pacific region to join together and build consensus for action; (2) the second forum will be held in Kuala Lumpur on 4 May 2008 at the time of the 11th Asian Pacific Congress of Nephrology (APCN). Zaki Morad, President of the 11th APCN, will host the second forum; (3) the International Organising Committee (IOC) of the 1st AFCKDI will continue its function by adding other experts, including the organisers of the APCN; (4) the AFCKDI is not an organisation by itself, nor does it belong to any society, but is organised by each host national society of nephrology. The IOC will assist the domestic committee for the success of the forum and will assure the continuation of the mission; (5) in order to organise the forum and promote CKD initiatives in the Asia Pacific region, the AFCKDI will look for support by both national and international societies. The AFCKDI will keep an intimate and mutual relation with the ISN, APSN and KDIGO.

Keywords Chronic kidney disease - AFCKDI . Asia $\cdot$ APSN $\cdot$ KDIGO · ISN COMGAN · Japan

\section{Introduction}

Nearly $50 \%$ of the global population lives in the Asian Pacific region, including the world's two large and most populous countries, China and India, which together account for over $35 \%$, and are the two countries with the highest incidence and prevalence of chronic kidney disease (CKD) dialysis patients (CKD 5-D). Recognising the need for a coordinated regional approach, the Japanese Society of Nephrology, as part of its 50th anniversary celebrations, established the Asian Forum on Chronic Kidney Disease Initiative (AFCKDI) in 2007. Aided by the International Society of Nephrology (ISN), Kidney Disease: Improving Global Outcomes (KDIGO), the Asian Pacific Society of Nephrology, the Australian and New Zealand Society of Nephrology and the Malaysian Society of Nephrology, two regional meetings have now been held: in Hamamatsu, Japan, in 2007 and in Kuala Lumpur, Malaysia, in 2008. The tasks facing AFCKDI are formidable, with enormous economic, cultural and geographic differences characterising the region. However, regional and international interest and support have been overwhelming. At very short notice, in Hamamatsu 16 countries submitted 56 abstracts, from which many were chosen to supplement the invited speakers, allowing representation of a very wide range of nations.

In Hamamatsu the agreed aims were to clarify the current state of $\mathrm{CKD}$ in the Asian Pacific region and to promote coordination, collaboration and integration of initiatives to combat this disease burden. As host chair, Dr. Seichi Matsuo introduced the three main topics for discussion: (1) CKD screening and early detection, (2) clinical practice guidelines (CPGs) and their implementation, and (3) education, implementation and international and regional cooperation and support.

\section{Screening for CKD}

Japan (S. Matsuo)

Statutory urinalysis has been carried out on industrial workers since 1972, school children since 1973 and persons aged over 40 years since 1982 [1]. Despite this, Japan unfortunately still ranks among the highest in the world for CKD-5D prevalence and incidence, with particularly a rising incidence of diabetic patients [2]. Clearly screening alone has made little impact, hence the Japanese Association of CKD has now been established and government funded to pursue a strategic research project aimed at prevention of CKD, or reducing CKD-5D.

Hong Kong (P. KT. Li)

In 2004 the ISN held a Consensus Workshop on Prevention of Progression of Renal Disease in Hong Kong [3]. The consensus was that screening for CKD was worthwhile in diabetic and hypertensive patients and in the relatives of patients with CKD due to diabetes, hypertension and glomerulonephritis, and that CKD was more common in individuals over 60-65. This consensus meeting published recommendations for prevention of progression once $\mathrm{CKD}$ was detected [4].

\section{Clinical practice guidelines and international collaboration}

\section{KDIGO (N. Lameire)}

A non-profit foundation governed by an international board of directors (six currently from our region), KDIGO aims to improve global CKD care by promoting, integrating and 
aiding implementation of CPGs [5], [6]. KDIGO has published a revision of the definition and classification of CKD [7], reviewed definition, evaluation and classifications in CKD mineral and bone disorders [8], and is in the process of preparing CPGs on hepatitis C in CKD [9]. KDIGO aims to provide reviews of the evidence behind clinical care and CPGs, allowing local carers to construct the own CPGs without the resource burden of this task. It also hopes to coordinate CPG development to prevent redundancy of effort and stimulate consensus (http://www.kdigo.org/).

CARI (R. Walker)

CARI is the only Asia Pacific regional group currently producing English language CPGs available on the web. The key aspects are an absolute need for a good evidence base to construct CPGs and the recognition that implementation must be inherent in the process [10].

\section{ISN (W. Couser)}

The ISN Commission on Global Advancement of Nephrology (ISN-COMGAN) pointed out the focus shifting from emphasis on renal replacement therapy to the "new nephrology"- the early detection and prevention of kidney disease and its cardiovascular consequences [4]. Core outreach programmes are encompassed under COMGAN [11]. The ISN Fellowship programme now emphasises training in clinical epidemiology and outcomes research. The ISN Continuing Nephrology Education (CNE) programme supports over 50 educational events each year, reaching over 10,000 health-care workers, with an emphasis on early detection and treatment of CKD. The restructured ISN Sister Centre programme supports 40 centre relationships worldwide aimed at progressing the developing centre through to becoming a regional, independent focus for promotion of all aspects of renal health care. The ISN Research and Prevention Committee has developed the programme for detection and management of CKD, hypertension, diabetes and cardiovascular diseases.

\section{Diversity and specificity of CKD in Asia}

Speakers dealt with CKD in the COMGAN regions, first from the two most populous countries, China and India, then a mix of developing and developed countries of differing sizes and economies. Highlighted was the urgent need to develop strategies to combat CKD, given the huge population of Asia, the high prevalence of CKD and the poor economic state of much of the region.
China (W. Chen)

A randomly selected population-based screening study in southern China (both rural and urban) showed $10.6 \%$ had proteinuria, haematuria or reduced estimated GFR. Independent risk factors were age, hypertension and diabetes.

India (V. Jha)

CKD, diabetes and hypertension have been identified as increasing in prevalence in several small surveys. Diabetes is the commonest cause of end-stage renal diseases (ESRD); 73\% of ESRD patients present less than 3 months before diagnosis [12].

Korea (H. J. Chin)

A nationwide survey from health checks in 39 hospitals indicated a prevalence of CKD stages 1, 2, 3 or more of $1.39,3.64$ and $2.67 \%$, respectively, with very similar risk factors to Western countries, and a particularly high prevalence in the elderly.

Nepal (S. K. Sharma)

In this country, where renal replacement therapy (RRT) cannot be afforded, a door-to-door screening and intervention programme was conducted. Of 3,218 people over 20, CKD was detected in $10.6 \%$. Age and diabetes were particularly predictive. When hyperglycaemia and hypertension were controlled, regression or stabilisation of proteinuria was seen in $52 \%$.

Japan (K. Iseki)

In 2005 Japan had the world's highest prevalence of CKD5 patients, 2,018 per million population (pmp) [13]. Sleep apnoea has recently been shown to be particularly common in Japanese CKD-5 patients, 30.5\% compared with a nonCKD-5 population prevalence of $15.1 \%$ [14].

Australia (D. Harris)

The AUSDIAB study [15] has indicated a population prevalence of CKD similar to other developed countries. Automatic reporting of estimated GFR (eGFR, modified MDRD formula) by laboratories, general practitioner education and screening/intervention studies are underway. A particularly important issue is "How can developed countries help developing nations?" Screening and intervention programmes in Indonesia and Brunei are being assisted by Australian centres. 
Screening, risk factors, evaluation, comorbidity and intervention in CKD in Asia

Many important issues were discussed, including: (1) Who should be screened? Cost effectiveness suggests a targeted approach. (2) What is the high-risk population? Is it similar to those in North America and Europe or different in Asia? (3) Is it necessary to study selected populations using epidemiological designs to collect regional data? (4) Is it necessary to have a common language about criteria for eGFR and urinary protein/albumin estimation in Asia? Is haematuria particularly relevant in Asia with the prevalence of glomerulonephritis, especially IgA disease? (5) Should we intervene in high-risk populations? Which subgroups would benefit most? What would be most cost-effective?

Estimating GFR in Asian populations

Standardised methods for estimating GFR are essential for detection and classification of CKD. The MDRD formula was not developed in Asian subjects, hence eGFR formulae need to be developed.

\section{China (L. Zuo)}

The broad issues for proper selection of eGFR formulae were introduced [16, 17, 18]. Methods for developing estimating equations were reviewed, including the inherent problems involved in regression, linear assumption and calibration of plasma creatinine or other measurements. Variations can lead to systemic differences in eGFR results. The recommendation was that eGFR should be developed based on both the ethnic group and the method and calibration of plasma creatinine or other measurements.

Japan (M. Horio)

The Japanese CKD Initiative has on-going studies to refine a Japanese eGFR equation [19-21]. eGFR by the MDRD formula was compared with inulin renal clearance in 247 Japanese CKD patients. Serum creatinine was measured by an enzymatic method in a central laboratory, which gave results virtually equivalent to standardised creatinine values. A tendency for eGFR MDRD to overestimate GFR was adjusted by introducing an ethnic coefficient ( $X 0.808$ ) for Japanese, calculated by minimising the sum of squared errors between eGFR MDRD and inulin clearances in patients with GFR $<90 \mathrm{ml} / \mathrm{min} / 1.73 \mathrm{~m}^{2}$.

China (Y.-C. Ma)

The Chinese eGFR Collaboration Group has produced a modified EGFR for Chinese $\left(\mathrm{eGFR}=175 \times \mathrm{Pcr}^{-1.234} \times\right.$ age $^{-0.179} \times 0.79$ for females). Changes in eGFR with ageing were studied in 747 apparently healthy Chinese subjects [22]. Jaffe's method was used in a central laboratory to measure serum creatinine. eGFR decrease per 10 tears was $4.3 \mathrm{ml} / \mathrm{min} / 1.73 \mathrm{~m}^{2}$, and about one-third of subjects 70 years or over had eGFR less than $60 \mathrm{ml} / \mathrm{min} /$ $1.73 \mathrm{~m}^{2}$. Overestimation of renal disease was a risk in the elderly. The utility of single or repeated spot urine albumin/creatinine ratios was studied in 659 Beijing residents (F. Wang). While microalbuminuria was present in $10.2 \%$ initially, this declined to $6.4 \%$ when repeated 4 months later, indicating that repeated measurements are needed to confirm CKD.

Prevalence, risk factors and comorbidity of CKD in Asia

Table 1 summarises the prevalence of CKD and prevalence/incidence of ESRD (RRT) reported in this meeting. Data were presented from 8 countries-Bangladesh, China, Malaysia, Mongolia, Sri Lanka, Singapore, Taiwan and Vietnam-as well as 19 further posters, indicating CKD is a major problem in all these countries, with some unique regional differences. These contained recurrent themes of increasing incidences of diabetes as a cause of ESKD and the need for early intervention schemes to combat the epidemic of ESKD in Asia, rather than the unaffordable alternative of RRT. All abstracts are available on the AFCKDI web site (http://www.jsn.or.jp/ AFCKDI2007/), or as published papers [23-25, 26, 27, 28, 29].

\section{Southern China (U. Kuok)}

In Macau, preliminary analysis from over 1,000 people indicates some evidence of CKD in over $20 \%$, but only $3-$ $5 \%$ have stages 3-5. However, in persons aged 65 years or over, this rises to more than $20 \%$.

\section{Southern Taiwan (H. C. Chen)}

Screening of family members of nearly 200 haemodialysis patients showed a $13 \%$ prevalence of eGFR $60 \mathrm{ml} / \mathrm{min} /$ $1.73 \mathrm{~m}^{2}$ and $17 \%$ prevalence of albuminuria. Only $15 \%$ showed awareness of CKD, indicating the need for more screening and education of family members [30, 31].

\section{Bangladesh (H. U. Rashid)}

A rural survey has indicated a prevalence of CKD of $17 \%$ in this country where RRT cannot be afforded. The need for primary care of CKD patients was highlighted [2]. 
Table 1 Prevalence of CKD and prevalence/incidence of ESRD (RRT)

\begin{tabular}{|c|c|c|c|c|c|c|c|}
\hline Area & $\begin{array}{l}\text { CKD prevalence } \\
\text { (stages) }\end{array}$ & GFR equation $^{c}$ & $\begin{array}{l}\text { Study } \\
\text { population }\end{array}$ & $\begin{array}{l}\text { Study } \\
\text { year }\end{array}$ & $\begin{array}{l}\text { ESRD } \\
\text { (incidence) }\end{array}$ & $\begin{array}{l}\mathrm{RRT} \\
\text { (prevalence) }\end{array}$ & Author \\
\hline Guangzou/Zhuhai & $10.6 \%(\mathrm{I}-\mathrm{V})$ & Classic MDRD & 4,642 & 2007 & NA & NA & W. Chen \\
\hline Korea & $1.39 \%$ (I), $3.64 \%$ (II), $2.67 \%$ (III-V) & Classic MDRD & 329,581 & 2005 & $185 \mathrm{pmp}^{\mathrm{a}}$ & $942 \mathrm{pmp}^{\mathrm{a}}$ & H. J. Chin \\
\hline Nepal & $10.6 \%(\mathrm{I}-\mathrm{V})$ & Classic MDRD & 3,218 & 2006 & Very few & Very few & S. K. Sharma \\
\hline Japan & $9.2 \%(\mathrm{III}-\mathrm{V})$ & $0.808 X_{M D R D}{ }^{\mathrm{d}}$ & 574,023 & 2006 & $275 \mathrm{pmp}^{\mathrm{a}}$ & $1,956 \mathrm{pmp}^{\mathrm{a}}$ & E. Imai \\
\hline Macau & $18.0 \%$ (I-II), $3.3 \%$ (III-V) & Classic MDRD & 1,047 & 2006 & NA & $933 \mathrm{pmp}$ & U. Kuo \\
\hline Taiwan & $6.9 \%(\mathrm{III}-\mathrm{V})$ & Classic MDRD & 6,001 & 2006 & $418 \mathrm{pmp}^{\mathrm{a}}$ & $2,226 \mathrm{pmp}^{\mathrm{a}}$ & C.C. Hsu \\
\hline Bangladesh & $17 \%$ in rural area & CG & & & $9 \mathrm{pmp}^{\mathrm{a}}$ & $92 \mathrm{pmp}^{\mathrm{a}}$ & H. U. Rashid, \\
\hline Mongolia & NA & NA & NA & 2005 & $(196 \mathrm{pmp})^{\mathrm{b}}$ & $36 \mathrm{pmp}$ & K. Gelegjamts \\
\hline Singapore & $4.45 \%$ (III-V) & Classic MDRD & 2,112 & & NA & NA & B. W. Teo \\
\hline Vietnam & $3.9 \%(\mathrm{III}-\mathrm{V})$ & Classic MDRD & 8,509 & & NA & NA & J. Ito \\
\hline Beijing & $9.3 \%(\mathrm{I}-\mathrm{V}), 1.7 \%(\mathrm{III}-\mathrm{V})$ & $1,23 \mathrm{XMDRD}^{\mathrm{d}}$ & 13,925 & & NA & NA & L. Zhang \\
\hline Bhopal & $3.2 \%($ age $>60$, DM $58.4 \%)$ & Classic MDRD & 572,029 & 2001 & NA & $152 \mathrm{pmp}$ & V. Jha \\
\hline Indonesia & $5.8 \%$ (I), $7.0 \%$ (II) $5.2 \%$ (III-V) & CG & 6,040 & 2006 & NA & NA & Dharmeizar \\
\hline Australia & NA & NA & & 2006 & $115 \mathrm{pmp}^{\mathrm{a}}$ & $778 \mathrm{pmp}^{\mathrm{a}}$ & USRDS \\
\hline Malaysia & NA & NA & & 2006 & $119 \mathrm{pmp}^{\mathrm{a}}$ & $615 \mathrm{pmp}^{\mathrm{a}}$ & Z. Morard \\
\hline Thailand & NA & NA & & 2006 & $139 \mathrm{pmp}^{\mathrm{a}}$ & $286 \mathrm{pmp}^{\mathrm{a}}$ & K. Praditpornsilpa \\
\hline HongKong & NA & NA & & 2006 & $140 \mathrm{pmp}^{\mathrm{a}}$ & $994 \mathrm{pmp}^{\mathrm{a}}$ & USRDS \\
\hline Shanghai & NA & NA & & 2006 & $282 \mathrm{pmp}^{\mathrm{a}}$ & $447 \mathrm{pmp}^{\mathrm{a}}$ & USRDS \\
\hline
\end{tabular}

Results in this table were obtained from the reports of this conference and published studies. Some of the data may be different from the data published later

NA not available, $C G$ Cockcroft-Gault, pmp per million people, $C K D$ chronic kidney disease, ESRD end-stage renal diseases

a Data were obtained from the USRDS database 2006 (http://www.usrds.org/)

${ }^{\mathrm{b}}$ Renal replacement therapy (RRT) was not applied to every patient

c Classic MDRD used an ethnic cofactor for non-black without creatinine standardisation

${ }^{\mathrm{d}}$ Only Chinese and Japanese data used an ethnic cofactor (1.23 and 0.808, respectively) for the MDRD equation with creatinine standardisation

\section{Mongolia (K. Gelegjamts)}

There are unique local issues in this isolated country. A survey of hospitalised patients from 2002-2005 showed a high incidence of CKD because of nephrolithiasis, particularly in children and women. Kidney and urinary tract infection was the third commonest cause of illness in the general community, and the commonest cause of hospital morbidity. Chronic pyelonephritis and glomerulonephritis are the main causes of ESRD, contributed to by the harsh climate, high fertility rate and poverty.

\section{Sri Lanka (G. Priyadarshana)}

In the north-central and western provinces (Polonnaruwa and Anuradhapura), there is a very high prevalence of a chronic interstitial disease of unknown cause. In Anuradhapura, CKD is the leading cause of in-hospital mortality. Environmental toxins are suspected, but have not been identified. Elsewhere in Sri Lanka, the causes of ESRD are similar to other counties.
Singapore (B. W. Teo)

Of over 200 persons presenting to one academic hospital for voluntary health screening, only $1.6 \%$ had a serum creatinine above the normal range, but $4.5 \%$ had CKD stage 3-5 when eGFR was calculated.

\section{Malaysia (Z. Morad)}

Malaysia has seen a rapid rise in ESRD because of diabetes in the last 2 decades, such that by 2006 it was the cause of $57 \%$ of ESRD, the highest in the world, mirroring the high $(11.50 \%)$ community incidence of diabetes. Glomerulonephritis and stone disease are falling as causes of ESRD.

\section{Vietnam (J. Ito)}

Japan has collaborated with Vietnam to find a prevalence of CKD stages 3-5 in 4\% and hypertension $>30 \%$ in 8,500 subjects aged $>40$ years in one region [32]. 


\section{Intervention in CKD in Asia}

Various attempts to improve care

in CKD were presented

\section{Taiwan (S. L. Wang)}

The Kaohsiung Medical University Hospital led a national care project starting in 2003. About 1,400 patients with CKD stage 3-5 have been enrolled. The investigators goals were for more CKD patients to choose home peritoneal dialysis over centre haemodialysis (result, marginal fall), an increase in patients on rHuEPO (result, 68.8-83.0\%) and permanent vascular access (result, 38.5-63.0\%), higher hematocrits (result 23.9-25.2\%) and reduced hospitalisation rates before initiation of dialysis. The programme was successful for most of the goals, though the proportion of patients choosing PD as the primary treatment modality fell marginally. The authors concluded that an integrated CKD care programme is effective in improving the dialysispreparedness and clinical profile of CKD patients. The message was in addition to steps needed to slow disease progression; CKD care should also include preparing patients for renal replacement therapy.

\section{Indonesia (Dharmeizar)}

The utility of a questionnaire-based screen for CKD risk factors with blood pressure and urinalysis was assessed in four rural areas of Indonesia. Of 6,040 subjects with a mean age 41 years, $41 \%$ had obesity, $14 \%$ hypertension, $22 \%$ diabetes and $3.6 \%$ proteinuria; 1,100 had serum creatinine measured, resulting in a $5.7 \%$ prevalence of CKD. The high incidence of obesity was a surprise, and in general the results suggest that this approach needs to be viewed with caution, since most measurements were performed only once.

\section{Japan (S. Matsuo)}

The outcomes from the Japanese Governmental Programme of Urinalysis commenced in 1973 were reported $[28,29]$. Urinalysis is carried out in population groups, particularly school children, employees and all citizens over 40 years of age. It is mandatory in the first two groups, and about $44 \%$ of the last group have been tested. Urinary abnormalities were noted in $2.7,6.8,4.9,6.3$ and $18.4 \%$ of elementary school students, junior high school students, high school students, industry workers and citizens over 40 years of age, respectively. Despite a decline in the contribution of glomerulonephritis (GN) to ESRD, the overall prevalence of ESRD in Japan has been relentless, and the numbers have been constantly increasing. The mean age of new Japanese ESRD patients with GN showed a significantly faster increase than in US patients, whereas those of patients with diabetes or nephrosclerosis increased at the same rate. It appears that while the urine testing programme has made a positive difference in $\mathrm{GN}$, it has had little impact on the overall growth of ESRD, possibly because the new lifestyle diseases and population age more than compensated for the decline in GN cases. Nevertheless, the database that has been accumulated as a result of the screenings is a fantastic one and can be mined to get valuable data of a type probably not available anywhere else in the world [1].

\section{Mission and future action plan}

The burden of CKD is high and unique in each of our neighbours. There is a clear need for coordination, collaboration and integration of initiatives to fight the epidemic of CKD in the Asian Pacific region; however, there is a considerable amount of variability in the resource availability among different countries or regions. Access to global information and evidence databases is also limited in some. To overcome these limitations, it was agreed that AFCKDI could play a very valuable role in harmony with ISN (especially COMGAN activity) and APSN activity, and we should continue to embrace the opportunity in the form of this meeting further in the future. There is no question that this is also a very good opportunity to give strength to networks and friendship of nephrologists in our region.

Few countries have developed local evidence-based clinical practice guidelines (CPGs) for CKD. Fortunately, global CKD guideline development is now in progress, and the definition and classification system introduced by KDIGO has been well accepted in this area. However, several local issues need to be addressed.

These include (1) estimated GFR equation(s) based on standardised creatinine estimation, which most efficiently reflect the Asian ethnicities, (2) efficient screening methods, which reflect the common pathogenesis of CKD in Asian countries, and (3) short-term strategies for intervention.

The ISN-KHDC programme for delaying progression could be applied in most of Asia areas regardless of economic status. Availability of interventions in other co-morbidities and complications of CKD, such as renal anaemia and CKD-MBD (mineral bone disease), varies among countries and regions because of economic status and/or public health policy.

We also need to facilitate collaboration, coordination and integration of locally developed CPGs, aiming to resolve the gaps in clinical practice. There is substantial 
room for cooperation in implementing CPGs in the regions where resources are limited.

There are good examples of corporation between developed and developing countries. We need to expand this effort not just between two countries, but also among multiple relationships in our area by utilising the available resources of developed nations.

ESRD is a very visible outcome of CKD, and the availability of RRT is drastically different among countries and regions in the Asian Pacific area. Many lives are still lost because of lack of access to RRT. An international registry of patients on RRT among multiple countries in our area would be valuable.

Care of dialysis and renal transplant recipients can also be improved by implementing locally applicable global CPGs. More attention should be paid to previous live donors for renal transplantation because of the possible risk of future CKD.

The future plan for AFCKDI was decided as follows: (1) The AFCKDI will provide opportunity annually or biannually for every person who promotes CKD initiatives in the Asian Pacific region to join together and build consensus for action. (2) The second meeting was held at Kuala Lumpur in 2008, hosted at the 11th Asian Pacific Congress of Nephrology (APCN) by Zaki Morad, President of the 11th APCN. (3) The International Organising Committee (IOC) of the AFCKDI will continue its function by adding other experts, including the organiser of the next meeting. (4) The AFCKDI is not an organisation by itself nor does it belong to any society. Meetings will be organised by each host national society of nephrology. The IOC will assist the domestic committee for the success of the forum and will assure the continuation of the mission. (5) In order to organise the forum and promote CKD initiatives in the Asian Pacific region, the AFCKDI will look for support by both national and international societies. The AFCKDI will keep an intimate and mutual relation with the ISN, APSN and KDIGO.

Finally, we have reached the following consensus as the mission of the AFCKDI and decided on the continuation of this effort in the future: (1) to develop a consensus as a protocol of CKD detection in our region; (2) to analyse risk factors and cost-effective evaluation of the intervention; (3) to establish a network on the CKD Initiative in our region; (4) to contribute to the global initiative by using resources in our region.

Acknowledgments The AFCKDI 2007 was organised by the JSN and also supported by funds from the APSN, ISN-CME and the Australian New Zealand Society of Nephrology. The authors express sincere thanks to every participant in this forum for their enthusiasm and passionate discussion. Every abstract and the list of participants are available on the website http://www.jsn.or.jp/AFCKDI2007/ index.html.

\section{Appendix}

Organization

President: Akira Hishida (President, JSN).

Secretary General: Yusuke Tsukamoto, Secretary: Yoshinari Yasuda.

International Organizing Committee.

Haiyan Wang (Co-chair), Yusuke Tsukamoto (Cochair), Gavin Becker, Evan Lee Jon Choon, Hung-Chun Chen, Dae-Suk Han, Vivekanand Jha, Philip KT Li, Kriang Tungsanga, and Rowan Walker.

Domestic Organizing Committee:

Seiichi Matsuo (Chair), Kunitoshi Iseki (Co-chair), Tadao Akizawa, Yasuhiro Ando, Masafumi Fukagawa, Yasuhiko IIno, Takashi Igarashi, Hiroyasu Iso, Iekuni Ichikawa, Sadayoshi Ito, Yuhei Ito, Daijo Inaguma, Enyu Imai, Hirokazu Imai, Shunya Uchida, Nobuyuki Ura, Masayuki Endo, Kazo Kaizu, Naoki Kashihara, Yutaka Kiyohara, Yasuhiko Tomino, Ichiei Narita, Kosaku Nitta, Masakazu Haneda, Shigeko Hara, Hideki Hirakata, Masaru Horio, Hirofumi Makino, Takeshi Matsuyama, Toshio Miyata, Toshiki Moriyama, Kunihiro Yamagata, Kenji Wakai, Tsuyoshi Watanabe.

Hosted by the Japanese Society of Nephrology.

Affiliated by the Asian Pacific Society of Nephrology, the International Society of Nephrology-COMGAN, the KDIGO/Kidney Disease: Improving Global Outcomes.

\section{References}

1. Imai E, Yamagata $\mathrm{K}$, Iseki $\mathrm{K}$, Iso $\mathrm{H}$, Horio $\mathrm{M}$, Makino $\mathrm{H}$, et al. Kidney disease screening program in Japan: history, outcome, and perspectives. Clin J Am Soc Nephrol. 2007;2:1360-6.

2. Nakai S, Wada A, Kitaoka T, Shinzato T, Nagura Y, Kikuchi K, et al. An overview of regular dialysis treatment in Japan (as of 31 December 2004). Ther Apher Dial. 2006;10:476-97.

3. Li PK, Weening JJ, Dirks J, Lui SL, Szeto CC, Tang S, et al. A report with consensus statements of the International Society of Nephrology 2004 Consensus Workshop on Prevention of Progression of Renal Disease, Hong Kong, June 29, 2004. Kidney Int Suppl 2005;94:S2-7.

4. Dirks JH, de Zeeuw D, Agarwal SK, Atkins RC, Correa-Rotter R, D'Amico G, et al. Prevention of chronic kidney and vascular disease: toward global health equity-the Bellagio 2004 declaration. Kidney Int Suppl. 2005;98:S1-6.

5. Eknoyan G, Lameire N, Barsoum R, Eckardt KU, Levin A, Levin $\mathrm{N}$, et al. The burden of kidney disease: improving global outcomes. Kidney Int. 2004;66:1310-4.

6. Levey AS, Atkins R, Coresh J, Cohen EP, Collins AJ, Eckardt $\mathrm{KU}$, et al. Chronic kidney disease as a global public health problem: approaches and initiatives-a position statement from Kidney Disease Improving Global Outcomes. Kidney Int. 2007;72:247-59.

7. Levey AS, Eckardt KU, Tsukamoto Y, Levin A, Coresh J, Rossert J, et al. Definition and classification of chronic kidney 
disease: a position statement from Kidney Disease: Improving Global Outcomes (KDIGO). Kidney Int. 2005;67:2089-100.

8. Moe S, Drueke T, Cunningham J, Goodman W, Martin K, Olgaard K, et al. Definition, evaluation, and classification of renal osteodystrophy: a position statement from kidney disease: improving global outcomes (KDIGO). Kidney Int. 2006; 69:1945-53.

9. Kidney Disease: Improving Global Outcomes. KDIGO clinical practice guidelines for the prevention, diagnosis, evaluation, and treatment of Hepatitis $\mathrm{C}$ in chronic kidney disease. Kidney Int 2008;73:S1-99.

10. Harris D, Thomas M, Johnson D, Nicholls K, Gillin A. The CARI guidelines. Prevention of progression of kidney disease. Nephrology (Carlton). 2006;11(Suppl 1):S2-197.

11. Dirks JH, Robinson SW. The global perspective of the International Society of Nephrology: a decade of experience with COMGAN. Kidney Int. 2005;68:1395-410.

12. Modi GK, Jha V. The incidence of end-stage renal disease in India: a population-based study. Kidney Int 2006;70:2131-3.

13. Nakai S, Masakane I, Akiba T, Iseki K, Watanabe Y, Itami N, et al. Overview of regular dialysis treatment in Japan (as of 31 December 2005). Ther Apher Dial. 2007;11:411-41.

14. Iseki K, Tohyama K, Matsumoto T, Nakamura H. High Prevalence of chronic kidney disease among patients with sleep related breathing disorder (SRBD). Hypertens Res. 2008;31:249-55.

15. White SL, Polkinghorne KR, Cass A, Shaw J, Atkins RC, Chadban SJ. Limited knowledge of kidney disease in a survey of AusDiab study participants. Med J Aust. 2008;188:204-8.

16. Coresh J, Astor BC, McQuillan G, Kusek J, Greene T, Van Lente $\mathrm{F}$, et al. Calibration and random variation of the serum creatinine assay as critical elements of using equations to estimate glomerular filtration rate. Am J Kidney Dis. 2002;39:920-9.

17. Zuo L, Ma YC, Zhou YH, Wang M, Xu GB, Wang HY. Application of GFR-estimating equations in Chinese patients with chronic kidney disease. Am J Kidney Dis. 2005;45:463-72.

18. Ma YC, Zuo L, Chen JH, Luo Q, Yu XQ, Li Y, et al. Modified glomerular filtration rate estimating equation for Chinese patients with chronic kidney disease. J Am Soc Nephrol. 2006;17:2937-44.

19. Imai E, Horio M, Iseki K, Yamagata K, Watanabe T, Hara S, et al. Prevalence of chronic kidney disease (CKD) in the Japanese general population predicted by the MDRD equation modified by a Japanese coefficient. Clin Exp Nephrol. 2007;11:156-63.

20. Imai E, Horio M, Nitta K, Yamagata K, Iseki K, Hara S, et al. Estimation of glomerular filtration rate by the MDRD study equation modified for Japanese patients with chronic kidney disease. Clin Exp Nephrol. 2007;11:41-50.
21. Imai E, Horio M, Nitta K, Yamagata K, Iseki K, Tsukamoto Y, et al. Modification of the modification of diet in renal disease (MDRD) study equation for Japan. Am J Kidney Dis. 2007; 50:927-37.

22. Ma YC, Zuo L, Chen JH, Luo Q, Yu XQ, Li Y, et al. Improved GFR estimation by combined creatinine and cystatin $C$ measurements. Kidney Int. 2007;72:1535-42.

23. Zhang L, Zhang P, Wang F, Zuo L, Zhou Y, Shi Y, et al. Prevalence and factors associated with CKD: a population study from Beijing. Am J Kidney Dis. 2008;51:373-84.

24. Zhang L, Zuo L, Wang F, Wang M, Wang S, Liu L, et al. Metabolic syndrome and chronic kidney disease in a Chinese population aged 40 years and older. Mayo Clin Proc. 2007; 82:822-7.

25. Zhang L, Zuo L, Xu G, Wang F, Wang M, Wang S, et al. Community-based screening for chronic kidney disease among populations older than 40 years in Beijing. Nephrol Dial Transplant. 2007;22:1093-9.

26. Zhang L, Zhao F, Yang Y, Qi L, Zhang B, Wang F, et al. Association between carotid artery intima-media thickness and early-stage CKD in a Chinese population. Am J Kidney Dis. 2007;49:786-92.

27. Thaha M, Widodo, Pranawa W, Yogiantoro M, Tomino Y. Intravenous $N$-acetylcysteine during hemodialysis reduces asymmetric dimethylarginine level in end-stage renal disease patients. Clin Nephrol 2008; 69:24-32.

28. Irie F, Iso H, Sairenchi T, Fukasawa N, Yamagishi K, Ikehara S, et al. The relationships of proteinuria, serum creatinine, glomerular filtration rate with cardiovascular disease mortality in Japanese general population. Kidney Int. 2006;69:1264-71.

29. Yamagata K, Iseki K, Nitta K, Imai H, Iino Y, Matsuo S, et al. Chronic kidney disease perspectives in Japan and the importance of urinalysis screening. Clin Exp Nephrol. 2008;12:1-8.

30. Hsu CC, Hwang SJ, Wen CP, Chang HY, Chen T, Shiu RS, et al. High prevalence and low awareness of CKD in Taiwan: a study on the relationship between serum creatinine and awareness from a nationally representative survey. Am J Kidney Dis. 2006; 48:727-38.

31. Kuo HW, Tsai SS, Tiao MM, Yang CY. Epidemiological features of CKD in Taiwan. Am J Kidney Dis. 2007;49:46-55.

32. Ito J, Dung DT, Vuong MT, Tuyen do G, Vinh le D, Huong NT, et al. Impact and perspective on chronic kidney disease in an Asian developing country: a large-scale survey in north Vietnam. Nephron Clin Pract 2008;109:c25-32. 